\title{
Du marché unique à la monnaie unique LEA : une formation pratique
}

Approche par le biais de la maîtrise

\section{Émilie Clauzure}

\section{(2) OpenEdition}

\section{Journals}

Édition électronique

URL : http://journals.openedition.org/asp/2858

DOI : 10.4000/asp.2858

ISSN : 2108-6354

Éditeur

Groupe d'étude et de recherche en anglais de spécialité

Édition imprimée

Date de publication : 1 décembre 1998

Pagination : 261-281

ISSN : 1246-8185

\section{Référence électronique}

Émilie Clauzure, «Du marché unique à la monnaie unique LEA : une formation pratique », ASp [En ligne], 19-22 | 1998, mis en ligne le 29 mars 2012, consulté le 20 avril 2019. URL : http:// journals.openedition.org/asp/2858; DOI : 10.4000/asp.2858

Ce document a été généré automatiquement le 20 avril 2019

Tous droits réservés 


\title{
Du marché unique à la monnaie unique LEA : une formation pratique
} Approche par le biais de la maîtrise

\author{
Émilie Clauzure
}

1 Les filières LEA ont été créées, à un moment particulier, il y a environ une vingtaine d'années (1973-74 pour Limoges), alors que l'idée d'Europe, de marché unique européen, se dessinait avec plus de précision, et d'urgence, dans les esprits. À côté de la voie traditionnelle de l'enseignement des langues vivantes (Littératures, cultures étrangères LCE), s'ouvrait la possibilité d'un enseignement (d'une formation?) qui se voulait axé sur des besoins pratiques, appliqués, ou plus directement applicables, au monde de l'emploi : la filière Langues étrangères appliquées (LEA) (voir fig. 1). L'enseignement des langues était jusqu'alors limité à la littérature, à la linguistique et à la civilisation dispensant soit une formation humaniste, "désintéressée ", ne débouchant sur aucune perspective professionnelle, soit à la préparation aux concours de recrutement de professeurs du secondaire (CAPES, Agrégation), à la voie de la recherche par le biais des maîtrises, DEA et doctorats en particulier, avec pour débouchés quasi exclusifs une carrière dans l'Éducation nationale. L'université est là dans son rôle de reproduction à l'identique, formant dans l'un et l'autre cas des formateurs de ses futurs usagers, les uns à échéance immédiate, les autres à échéance différée. La création de LEA, en revanche, a correspondu à un désir et à un besoin de professionnalisation pour la société " civile ", avec le souci qu'existent à tout moment des passerelles et des possibilités de réorientation vers la filière dont elle se distinguait. 


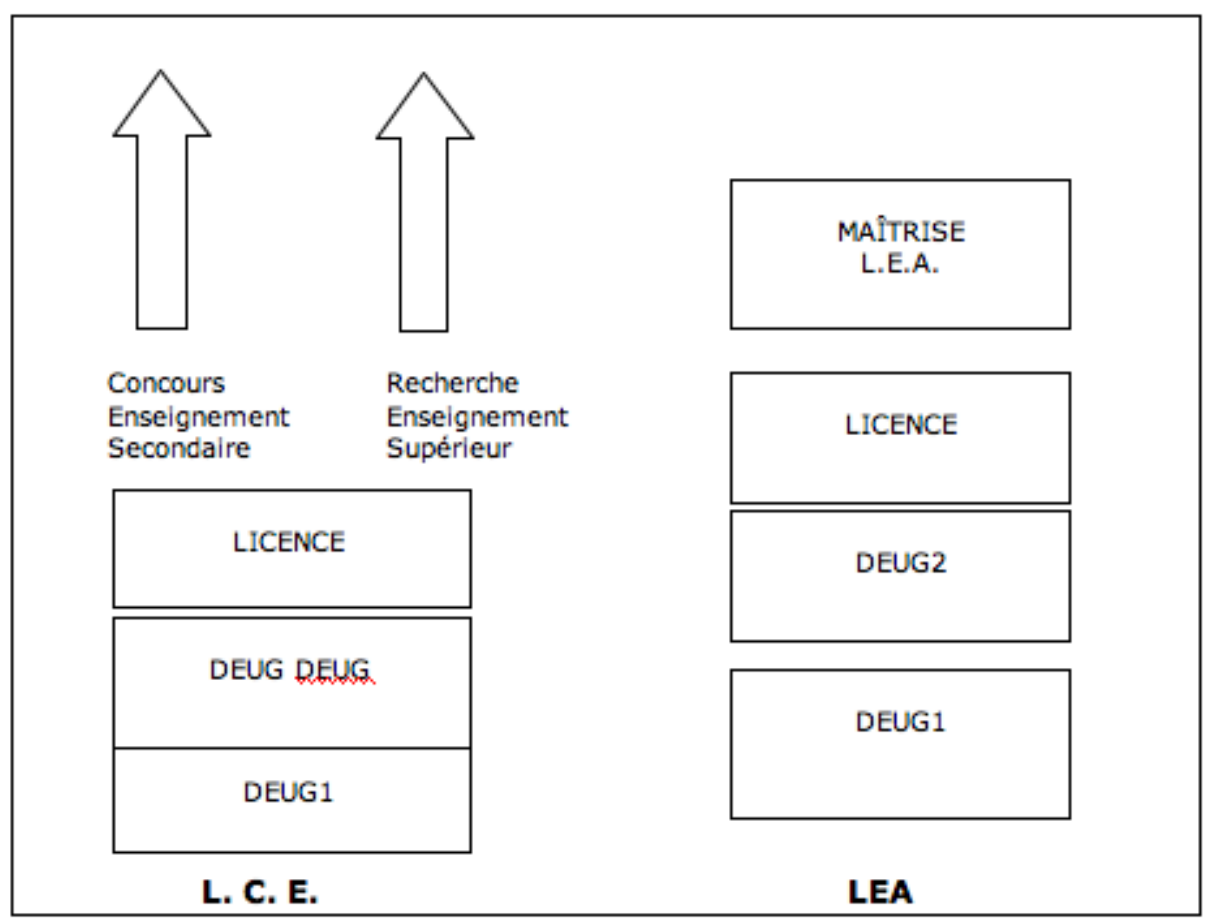

2 En raison de l'ouverture des frontières et l'internationalisation des échanges à l'horizon 2000, apparaissait, pensait-on, un besoin croissant de personnel qualifié sachant utiliser au moins deux langues étrangères dans des domaines d'application.

Pour les initiateurs locaux de LEA, avant de se projeter à l'extérieur, il semblerait que le souci premier ait consisté à se différencier d'abord de l'intérieur, c'est-à-dire de la voie LCE.

4 En s'appuyant sur ces données et ces intentions, mon projet cherche à vérifier dans quelle mesure, alors que la vingtième promotion de maitrise (1996-97) vient d'être enregistrée à la faculté des Lettres de Limoges, la volonté de formation pratique transparaît dans les stages et les sujets de mémoires, Travaux d'études et de recherche (TER) au terme du cursus, c'est-à-dire en quatrième et dernière année, appelée encore année de maîtrise. Les différentes générations d'étudiants suivent-elles fidèlement les directives officielles régissant la formation ou se laissent-elles aussi guider par les fluctuations de la conjoncture, s'inspirent-elles d'autres possibilités avantageuses dont elles peuvent bénéficier par ricochet?

5 Avant d'analyser le phénomène, il nous semble important de rappeler la spécificité de la formation telle qu'elle a été décrétée au départ, à savoir : le cahier des charges initiales où se marque l'originalité de la filière par rapport à LCE. Le concept d'études longues de cette dernière étant contrasté avec celui de la professionnalisation LEA (études réputées plus courtes) où quatre années au maximum, maîtrise incluse, sont envisagées pour le cycle :

- le choix de langues ;

- le volume annuel de l'enseignement;

- les disciplines proposées ;

- le poids relatif des stages et des cours à la faculté ; 
- le nombre d'étudiants en maîtrise par rapport au nombre d'inscrits en première année 4 ans plus tôt.

6 L'étude, qui n'est pas une enquête sur la totalité de la formation ne fait pas apparaître les redoublements; elle n'est pas non plus une enquête sur l'insertion professionnelle des étudiants, mais s'appuiera le cas échéant sur les résultats que des travaux de ce type ont livrés.

Ensuite, je m’intéresserai au nombre de mémoires rédigés en anglais ; à la répartition des sujets selon les domaines d'activité; à l'influence des accords inter-universitaires européens et de la présence des problèmes d'actualité dans le choix des sujets.

Enfin, il s'agira de voir

- l'adéquation entre le thème d'études et la vocation professionnelle de la filière, l'ampleur ou la limite de la réussite par rapport aux thèmes privilégiés ;

- le pourcentage d'étudiants entrant dans la vie active par rapport à ceux qui poursuivent des études, ce qui dépend de plusieurs éléments internes (sans parler des éléments externes, comme la conjoncture de l'emploi) ;

- la dimension pédagogique (origine et composition du corps enseignant);

- l'aspect financier (dotation d'organismes publics, privés locaux ou nationaux).

\section{Origines de la formation à l'Université de Limoges}

Les textes officiels réglementent la maîtrise et confèrent l'habilitation aux universités. Les trois principaux datent du 27 février 1973, du 16 janvier 1976 et du 7 juillet 1977 « relatif à la dénomination nationale de licence et de maîtrise de langues étrangères appliquées ». Ils fixent ses modalités d'instauration et d'application.

Le programme est dans ses grandes lignes resté inchangé depuis l'arrêté du 27 janvier 1981 article 3 (qui abrogeait celui du 7 juillet 1977) où le Ministère des Universités, DESUP, indiquait le profil de la formation de maîtrise. L'article 5 précisait [...] que «les universités peu(ven)t prévoir un autre stage au cours de l'année de maîtrise ».

Pour l'année universitaire 1997-98, il comporte un minimum de 375 heures d'enseignement réparties en deux groupes de matières, les langues et les mentions, ventilées en six modules distincts (LEA-Limoges 1997-98 : 1-2) (voir tableau 1).

Tableau 1. Modules LEA Limoges

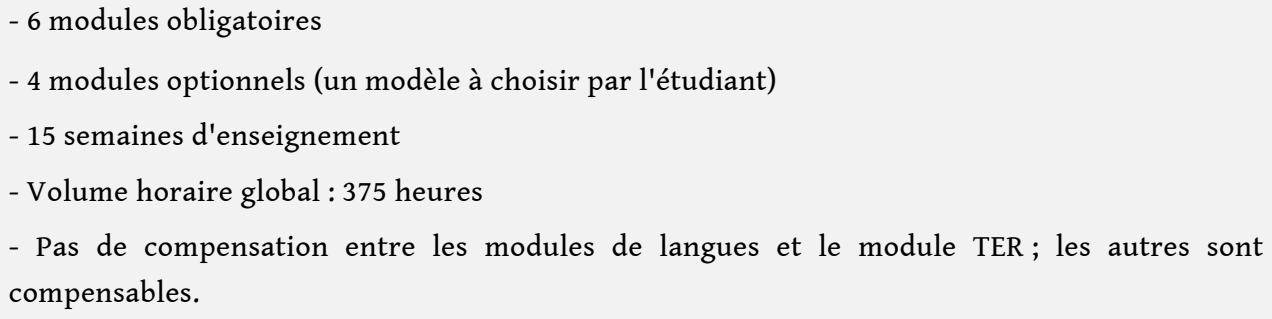

Pour les autres dispositions relatives à la maîtrise, il faut observer que sur les six modules requis, le module Stage à l'étranger, à lui seul, représente la même durée (un semestre) dans le cursus que les cinq autres modules réunis et qu'il ne procure des équivalences d'unité de valeur (ou crédits) que dans le cas d'un séjour universitaire. En revanche, il 
n'est pas validé en tant que tel (c'est-à-dire en termes de notes pour l'obtention du titre) dans le cas d'un stage d'entreprise. espagnol, est le plus souvent l'anglais: des $2 / 3$ à la fin des années 1980 (espagnol et allemand se partageant le dernier $1 / 3$ avec un net avantage pour l'espagnol), la proportion est maintenant de $3 / 4$ avec encore quelques mémoires en espagnol et de très rares en allemand (de moins en moins d'étudiants partant en stage ou en échanges universitaires en Allemagne).

21 Il nous paraît utile de signaler l'évolution du taux d'accès en maîtrise, c'est-à-dire le pourcentage entre le nombre de maîtrises décernées et le flux d'entrée en $1^{\text {re }}$ année quatre ans plus tôt, étude en cohorte (voir tableau 2). 
Tableau 2. Évolution du taux d'accès en maîtrise

\begin{tabular}{|c|c|c|c|c|}
\hline $\begin{array}{l}\text { Années } \\
\text { DEUG1 }\end{array}$ & $\begin{array}{l}\text { Nombre } \\
\text { inscrits }\end{array}$ & $\begin{array}{l}\text { Année } \\
\text { maîtrise }\end{array}$ & $\begin{array}{l}\text { Nbre maîtrises } \\
\text { décernées }\end{array}$ & $\begin{array}{l}\text { \%.reçus.maîtrise./.inscrits DEUG1-4ans } \\
\text { + tôt }\end{array}$ \\
\hline $1974-75$ & 22 & $1977-78$ & 03 & 14 \\
\hline $1975-76$ & 46 & $1978-79$ & 04 & 09 \\
\hline 1976-77 & 59 & $1979-80$ & 11 & 19 \\
\hline $1977-78$ & 100 & $1980-81$ & Chiffres non connus & Chiffres non connus \\
\hline 1978-79 & 107 & 1981-82 & 08 & 07 \\
\hline $1979-80$ & 135 & $1982-83$ & 12 & 09 \\
\hline $1980-81$ & 138 & 1983-84 & 23 & 17 \\
\hline $1981-82$ & 140 & 1984-85 & 17 & 12 \\
\hline $1982-83$ & 170 & $1985-86$ & 11 & 06 \\
\hline $1983-84$ & 150 & $1986-87$ & 17 & 11 \\
\hline 1984-85 & 159 & $1987-88$ & 20 & 13 \\
\hline $1985-86$ & 174 & 1988-89 & 10 & 06 \\
\hline $1986-87$ & 176 & $1989-90$ & 13 & 07 \\
\hline $1987-88$ & 192 & $1990-91$ & 16 & 08 \\
\hline 1988-89 & 254 & 1991-92 & 20 & 08 \\
\hline $1989-90$ & 262 & $1992-93$ & 19 & 07 \\
\hline $1990-91$ & 230 & 1993-94 & 24 & 10 \\
\hline $1991-92$ & 245 & 1994-95 & 11 & 04 \\
\hline $1992-93$ & 261 & $1995-96$ & 08 & 03 \\
\hline $1993-94$ & 177 & 1996-97 & 11 & 06 \\
\hline
\end{tabular}

22 C'est en 1980-81 que le pourcentage est le plus élevé : 16,67\%. Depuis 1991-92, le pourcentage oscille autour de $10 \%$ c'est-à-dire, uniquement en termes d'accès à un niveau, des résultats deux fois supérieurs à la moyenne nationale. 
Rappelons en effet qu'au niveau national, 82966 maîtrises (y compris de sciences et techniques) ont été délivrées en 1995 pour 1692000 étudiants inscrits en $1^{\text {re }}$ année en 1991, soit 4,9 \% (MENRT1997b).

D'autre part la maitrise, dans la logique d'une filière appliquée, peut être considérée comme relevant d'études longues: il n'est donc pas étonnant qu'un nombre limité d'étudiants s'y inscrive. Plus qu'un pourcentage de réussite, ou d'échec, il faut y voir la preuve que la majorité des étudiants de facultés des Lettres et sciences humaines s'arrête à la fin du premier cycle ; qu'au niveau du deuxième, les pourcentages passent au-dessous de la barre des $10 \%$ et que le troisième cycle ne concerne qu'une poignée d'élus universitaires et sociaux.

Ces indications permettent de mieux éclairer le taux de réussite en maîtrise, c'est-à-dire le rapport entre le nombre d'inscrits et le nombre de maîtrises effectivement décernées. Une comparaison sur 20 ans entre le nombre d'inscrits en maîtrise et le nombre de titres décernés permet d'avoir une idée plus nette des résultats enregistrés (voir tableau 3).

Tableau 3. Nombre d'inscrits en maîtrise et titres décernés

\begin{tabular}{|c|c|c|c|}
\hline Années & Inscrits maîtrise & Maîtrises décernées & \% reçus maîtrise/ inscrits maîtrise \\
\hline $1977-78$ & 06 & 03 & 50 \\
\hline 1978-79 & 09 & 04 & 44 \\
\hline $1979-80$ & 14 & 11 & 78 \\
\hline $1980-81$ & 14 & chiffres non connus & chiffres non connus \\
\hline $1981-82$ & 14 & 08 & 57 \\
\hline 1982-83 & 33 & 12 & 36 \\
\hline 1983-84 & 35 & 23 & 65 \\
\hline $1984-85$ & 36 (dont 1 en alld-esp) & 17 & 47 \\
\hline $1985-86$ & 26(dont 1 en alld-esp) & 11 & 42 \\
\hline $1986-87$ & 37 & 17 & 45 \\
\hline $1987-88$ & 42 & 20 & 47 \\
\hline 1988-89 & 42 (dont 1 en alld-esp) & 10 & 23 \\
\hline $1989-90$ & 48 (dont 2 en alld-esp) & 13 & 27 \\
\hline $1990-91$ & 61 (dont 1 en alld-esp) & 16 & 26 \\
\hline 1991-92 & 64 & 20 & 31 \\
\hline 1992-93 & 51 & 19 & 37 \\
\hline
\end{tabular}




\begin{tabular}{|l|l|l|l|}
\hline $1993-94$ & 63 & 24 & 38 \\
\hline $1994-95$ & 50 & 11 & 22 \\
\hline $1995-96$ & 54 & 08 & 14 \\
\hline $1996-97$ & 55 & 11 & 20 \\
\hline
\end{tabular}

En 1977-78, première année d'ouverture de la maîtrise, un étudiant sur deux l'obtenait (50\%), en 1979-80, presque quatre sur cinq sont reçus $(78 \%)$, taux de réussite exceptionnel. En 1983-84, le pourcentage est toujours remarquablement élevé : $65 \%$. L'année suivante, les résultats avoisinent $50 \%$, proches de ceux de l'ouverture huit ans plus tôt. Depuis 1986, une stabilisation s'observe frôlant 40 \% en 1993-94.

De 1978 à 1997, soit 20 ans, 259 maîtrises au moins (la rédaction et la soutenance du mémoire sont des conditions sine qua non de l'obtention de la maîtrise) ont été décernées. Les résultats de troisdates précises nous semblent significatifs :

- en 1978, première année de fonctionnement de la maîtrise : 3 (2en anglais-allemandet 1 en anglais-espagnol);

- en 1987, à mi-parcours, dix ans après le lancement : 17 au total (8 en anglais-allemandet 9 en anglais-espagnol), enfin

- en 1996-97, dernière année en date : 11 (5 en anglais-allemandet 6 en anglais-espagnol). Voir tableau 4.

Tableau 4. Résultats maîtrise 1978, 1987 et 1997

\begin{tabular}{|l|l|l|l|}
\hline & 1978 & 1987 & 1997 \\
\hline Nombre maîtrises inscrites & $\mathbf{0 6}$ & 37 & 55 \\
\hline Angl-alld & 03 & 20 & 21 \\
\hline \hline Angl-espagnol & 03 & 17 & 34 \\
\hline Total maîtrises décernées & $\mathbf{0 3}$ & $\mathbf{1 7}$ & $\mathbf{1 1}$ \\
\hline Angl-alld & 02 & 08 & 05 \\
\hline Angl-espagnol & 01 & 09 & 06 \\
\hline
\end{tabular}

28 Alors que LEA est perçue comme une filière à majorité féminine (l'enquête de 1985 conduite à Limoges par LEA, l'ONISEP, le BUI et le Centre de calcul parlait d'une filière presque " essentiellement féminine »), les garçons opèrent une remontée en maîtrise. En effet, sur 235 titres de maitrise dont la scolarité a gardé la trace, 23 reviennent à des étudiants, soit $10 \%$, c'est-à-dire qu'il y aurait deux fois plus d'étudiants (qu'en DEUG) présents en maîtrise, au niveau de l'échelon le plus haut de la formation. 


\section{Les mémoires ou TER}

En principe, un exemplaire de chaque mémoire soutenu doit être déposé à la bibliothèque du département LEA, consigne qui fut respectée jusqu'en 1988-90. Faute d'archivage systématique depuis cette date, tant au niveau du département qu'au niveau de la scolarité, la consultation des documents requiert un travail d'enquête préalable auprès de collègues, des services de la faculté concernés ainsi que des vérifications croisées. Jusqu'en 1979, c'est-à-dire les deux premières années, les mémoires ont été rédigés indépendamment d'un séjour, ou plutôt sans séjour à l'étranger. À partir de 1980, les étudiants effectuent un stage d'entreprise ou un séjour linguistique (ce dernier étant la formule majoritairement choisie) de 4 mois, soit un semestre à l'étranger, le plus souvent dans une université partenaire de l'université de Limoges.

Les mémoires (Travaux d'études et de recherche) portent la marque de ce déplacement hors frontière.

Sur les 131 mémoires qui ont été examinés, la répartition par grands secteurs (du plus représenté au moins représenté) est la suivante :

- Économie : 58

- Administration ; politique locale ; politique nationale : 30

- Histoire locale ; Université : 22

- Questions sociales : 21

Figure 2. Répartition par secteur des TER

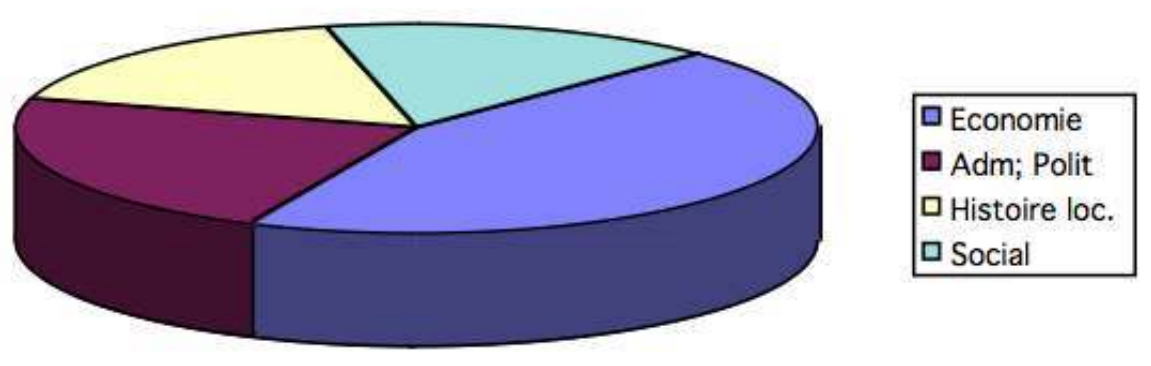

33 Une division plus fine permet de mieux voir comment ces secteurs sont ventilés en thèmes :

1. Agriculture \& industrie (secteurs primaire et secondaire) : 23

2. Questions sociales (emploi, chômage, questions féminines) : 21

3. Administration et politique locale : 19

4. Commerce \& tourisme : 15

5. Politique : 11

6. Histoire locale et patrimoine: 10

7. Université \& enseignement : 09

8. Économie \& environnement : 07

9. Media : 07

10. Finance : 03

11. Marketing : 03 


\section{Les thèmes}

L'étude des TER permet de constater que leurs thèmes obéissent à des constantes (ou régularités) qui, si elles n'apparaissent pas d'emblée, sont visibles lorsque l'ensemble des travaux est examiné. (Voir en annexes pour les titres des TER)

L'ancrage dans la région est très prégnant dès l'origine et le demeure. Avant l'instauration des stages à l'étranger, les expériences professionnelles avaient lieu localement le plus souvent et les TER reflétaient les préoccupations de la région. Elles n'ont pas disparu avec les déplacements en dehors de l'hexagone; au contraire, elles figurent en permanence au premier plan des sujets de réflexion

La région d'origine d'abord : l'agriculture restera un objet d'étude au fil des vingt années considérées, que ce soit l'agriculture française (locale, du Limousin) ou anglaise, dans sa structure propre ou par rapport aux contraintes européennes. L'autre activité locale traditionnelle, l'industrie de la porcelaine trouve sa place dans les TER, contrastée à la région anglaise avec laquelle le Limousin est jumelé, le Staffordshire.

L'apparition des régions d'accueil correspond strictement au moment où s'instaure le partenariat de l'université de Limoges avec les autres universités anglaises. Les dates s'échelonnent ainsi :

- 1983 : Portsmouth

- 1987 : Manchester

- 1988 : Coventry

- 1988 : Keele (Staffordshire)

- 1991 : Liverpool

- 1994 : Université de Caroline du Nord à Charlotte (États-Unis)

- 1996 : Aberdeen

Premier en date des partenaires, Portsmouth a suscité une vague de sujets de mémoires qui devait rester inégalée. On n'en dénombre pas moins de 21 , déclinés sous tous les registres, géographique, historique, industriel, architectural, universitaire, économique, social, religieux, colonial, sportif, touristique :

Une tendance semblable, mais bien atténuée, est visible pour Manchester quelques années plus tard, avec parfois une vision plus large.

L'histoire locale et le patrimoine qui peuvent se rattacher au même courant donnent lieu à 10 mémoires. Tous ont trait à la ville de Portsmouth et sa région. L'intérêt se porte aussi sur Bath, de ses origines romaines à nos jours.

1 Les autres mémoires se répartissent suivant les secteurs de l'économie, primaire: agriculture, pêche ; secondaire : industrie ; tertiaire : commerce, tourisme ; les questions sociales ; l'université ; la politique (nationale et internationale), et les médias.

Les questions sociales et leur détérioration pendant les années dites de thatchérisme suivent de près, devançant l'administration et la politique locales : l'inégalité, la police, les prisons, la condition des femmes, les personnes âgées, les jeunes, la taxe d'habitation ( community charge ou poll tax si controversée qu'elle fut abolie), les avatars et la dégradation de la notion de Sécurité sociale au cours des deux dernières décennies. 
43 Le monde universitaire, vu par le biais de l'établissement d'accueil, relève de la même volonté d'exploration des potentialités de la région. L'institution universitaire d'accueil d'abord, puis l'analyse de la vie étudiante en Grande-Bretagne, des difficultés économiques qui en découlent justifient quelques sujets. L'entrée de l'Université de Caroline du Nord à Charlotte comme partenaire d'échange croise enfin la dimension économique à celle de l'université comme moteur de la vie régionale.

44 Le monde de la presse, des médias et de la publicité en général ou appliqué à des cas particuliers a entraîné la rédaction de plusieurs mémoires.

45 L'intérêt pour les enjeux politiques, au niveau national et leurs conséquences, économiques, sociales ou proprement politiques, au niveau des relations internationales, surprend si l'on pense à la finalité de LEA. Il suscite 13 mémoires, allant de l'analyse de crises ponctuelles à des situations de conflit installées de longue date (conflit angloirlandais ou guerre civile anglo-irlandaise).

46 «La documentation technique » avait été lancée comme une possibilité de recherche au cours des deux premières années de maîtrise pour les étudiants restant à Limoges. La piste semblait prometteuse, jetant les prémisses de glossaires du transport en 1979, de la bourse et de l'export en 1980, mais elle s'est arrêtée brusquement .

47 L'examen des TER appelle une première remarque sur le faible nombre de ceux qui résultent d'un stage en entreprise. Lorsque c'est le cas, le TER traite d'un problème concret, fruit de l'observation directe, sensible dans le titre. Le plus exemplaire d'entre eux, en 1996, est peut-être celui qui a été réalisé dans l'industrie lourde des aciéries et qui s'intitule, Les profilés métalliques à froid en Grande-Bretagne (Long-cold rolled sections in the United Kingdom).

La remarque corollaire, c'est que la majorité d'entre eux, même s'ils intègrent économie ou gestion dans le libellé des sujets, traitent en fait de sujets de civilisation, au sens large telle que celle-ci est conçue en LCE (et le plus souvent en rapport avec les régions d'accueil).

49 L'interprétation de ces résultats n'est possible que si les TER sont mis en regard des stages qui les précèdent dans la chronologie du cursus. Le côté «académique», genre monographie de certains TER trouve alors son explication dans la nature du séjour effectué à l'étranger.

\section{Les stages : stage en entreprise ou séjour linguistique?}

Le programme de maîtrise rappelle l'obligation d'un séjour minimum de 4 mois à l'étranger (jusqu'en 1992-93, le stage à l'étranger était préconisé, mais non encore obligatoire), dans une Université ou dans une Entreprise (module Stage à l'étranger) et la rédaction d'un mémoire en langue étrangère (module TER). Latitude est laissée à l'étudiant LEA de choisir Université ou Entreprise, à la différence des stages dans les Écoles Supérieures de Commerce et pour l'obtention du Brevet de technicien supérieur. Aussi Assistant secrétaire trilingue, pour lesquels le Ministère (arrêté du 3 septembre 1997 : 80) indique clairement qu'il doit s'agir d'un stage en entreprise.

\section{Stage en milieu professionnel}

[...] Le candidat au brevet de technicien supérieur assistant secrétaire trilingue doit obligatoirement accomplir deux périodes de stage en entreprise à temps plein d'une durée de globale obligatoire de 12 semaines. Cette durée peut être prolongée 
de 4 semaines, à titre facultatif.

Ces stages se déroulent dans une ou plusieurs entreprises ou organismes ayant des activités internationales.

L'une des deux périodes aura obligatoirement lieu à l'étranger dans un pays non francophone. L'autre sera effectué soit à l'étranger, soit dans une entreprise (ou un organisme) située sur le territoire national mais dans un service ayant des relations avec l'étranger (et dans des langues autres que le français)

La mobilité géographique ne semble poser aucun problème : tous les étudiants concernés acceptent volontiers cette directive. Un tiers seulement optent pour un pays limitrophe de la France, les deux tiers s'égaillant dans le monde entier (pour moitié en Europe). Il faut noter que l'esprit international est bien développé, certains étudiants n'hésitant pas à s'aventurer jusqu'en Australie par le biais de postes d'assistants de langues.

Toutefois dans la pratique, le stage d'entreprise s'avère difficile à mettre en œuvre, tout d'abord pour des raisons purement structurelles. Indépendamment du dévouement et du talent (qui ne seront jamais reconnus à la hauteur de leurs mérites) des enseignants initiateurs du projet, il faut en effet souligner que la section, puis département LE, a été lancée sur des bases professionnalisantes avec des moyens physiques et matériels d'UFR d'anglais (qui sont si faire se peut encore plus limités que ceux de la faculté des Lettres). LEA n'avait pas un carnet d'adresses d'entreprises étrangères suffisant pour tous ses étudiants. Il incombait donc à ceux-ci de trouver par leurs moyens propres un stage en entreprise dans un autre pays. Le coût a longtemps constitué un second frein puissant, pour ne pas dire prohibitif, à l'essor d'un tel projet.

C'est pourquoi les stages à l'étranger rendus obligatoires à Limoges (avec cette réserve que les stages d'entreprise ne sont généralement pas ou fort peu défrayés) signifient, dans la pratique, un assouplissement de la formule.

Pour nombre d'étudiants, depuis la ratification d'accords inter-universitaires européens (en particulier, ERASMUS puis SOCRATES) et des dotations de mobilité afférentes (grâce auxquels les étudiants obtiennent des financements européens et régionaux), les stages se sont transformés, de fait, en séjours dans les universités. Pendant la décennie 1980, ceuxci ont représenté plus de $90 \%$ des séjours à l'étranger. L'engouement ainsi suscité prouve que ces programmes ont identifié un réel besoin de mobilité chez les étudiants mais aussi que ces derniers, avant même d'être engagés dans la vie professionnelle, ont bien perçu l'enjeu lié à l'ouverture des frontières et, dès que l'occasion leur en a été donnée, ils se sont hâtés de découvrir un autre pays en profondeur (où parfois même ils se sont installés).

Le mot stageassimilé à séjour linguistique prend dès lors un caractère ambigu. Certes, le séjour à l'université peut de plein droit être considéré comme un stage linguistique mais ne saurait plus en aucun cas recouvrir la notion de stage au sens de pratique professionnelle.

Ce glissement progressif de sens a suscité, à son tour, des consignes relatives au séjour linguistique, à savoir l'obligation pour les étudiants de s'inscrire à des cours dans l'université étrangère, de les suivre régulièrement et d'y subir des évaluations comptabilisables pour l'obtention de la maitrise. Il y obtient des notes qui sont validées à Limoges pour l'équivalent d'un ou de plusieurs cours, voire de modules complets. En Grande Bretagne, la plupart des étudiants valident le module d'anglais (comme en Allemagne, ils valident le module d'allemand) ainsi que des cours suivis dans d'autres langues vivantes, comme l'espagnol ou l'allemand et dans le domaine d'application. Il en 
va de même dans les autres pays; en Espagne, toutefois ils suivent très peu de cours d'espagnol. Au retour, l'étude des dossiers est faite cas par cas.

Les stages en entreprise ne sont pas réellement valorisés du strict point de vue des études. Pour l'administration, ils satisfont la seule obligation du séjour à l'étranger. Les étudiants ont cependant la possibilité de présenter un rapport de stage qui compte, dans le domaine d'application seulement, pour les points obtenus au-dessus de la moyenne.

Ainsi les étudiants désirant effectuer un stage en entreprise, d'abord dissuadéspar le coût et la nécessité où ils sont laissés d'agir seuls, se trouvent en outre du strict point de vue $\mathrm{du}$ rendement universitaire, pénalisés par rapport à leurs camarades optant pour les séjours linguistiques. Tenus d'être présents à temps plein dans l'entreprise, ils n'ont pas la possibilité de suivre les cours d'universités qui permettent à leurs camarades d'obtenir aisément les évaluations désormais exigées par l'établissement d'origine (cette situation pourrait même entraîner des risques de redoublement pour certains stagiaires d'entreprise). Leur mémoire qui a le mérite d'être axé sur un sujet technique (alors que les stagiaires de LEA étaient appréciés pour leurs nombreuses qualités, l'enquête de 1991-92 signalait que les compétences techniques demeuraient leur point faible)n'est l'objet d'aucune bonification spéciale : il est jugé exactement comme les autres TER de facture et d'inspiration beaucoup plus « livresques ». Les précisions qui viennent d'être fournies peuvent expliquer les réactions surprenantes au premier abord - à l'enquête de 1992-93 sur la question de l'utilité du stage de maîtrise pour l'insertion professionnelle (voir tableau 5).

Tableau 5. Utilité du stage d'entreprise pour l'insertion professionnelle, pourcentage de nonréponses

\begin{tabular}{|l|l|l|}
\hline 1982 & 1986 & 1993 \\
\hline $35 \%$ & $42 \%$ & $77 \%$ \\
\hline
\end{tabular}

En 1992-93, seulement $18 \%$ des étudiants de maîtrise pensaient que le stage était utile à l'insertion professionnelle. Il y eut $77 \%$ de non-réponses à la question de l'utilité des stages d'entreprise. Le commentaire donné par les analystes est le suivant :

ce qui résulte du fait que les étudiants ont effectué leur stage de maîtrise dans des universités étrangères et non dans des entreprises. (Renaudin $1984: 22)^{1}$

\section{Regain de faveur des stages en entreprise}

61 Jusqu'en 1994-95, seul un dixième de la promotion a opté pour les stages en entreprise. Deux caractéristiques intéressantes sont à noter lorsque c'est le cas. D'une part, les étudiants partent pour des destinations lointaines (Amérique latine, centrale : Venezuela, Équateur; Amérique du Nord) et, d'autre part, ce sont des établissements ou sociétés avec lesquels ils sont entrés en contact de leur propre initiative, par le biais de relations personnelles.

62 En dépit des facteurs dissuasifs qui viennent d'être indiqués, les étudiants semblent s'orienter désormais de manière plus significative vers les stages d'entreprise.

En 1996-97

63 L'augmentation de ceux-ci a été sensible en 1996. Cette année-là, sur 27 étudiants à l'étranger, si 17 effectuaient un séjour linguistique dans une université partenaire (dont 
un assistant en Australie), 9 étaient en stage d'entreprise partout dans le monde (certains, en raison de l'éloignement, ayant choisi d'allonger la durée du stage à 6 mois), c'est-à-dire un tiers des étudiants (voir tableau 6).

Tableau 6. Pays de stage en entreprise ou en université en 1996

\begin{tabular}{|c|c|c|c|c|c|}
\hline Pays & Allemagne & $\begin{array}{c}\text { Grande- } \\
\text { Bretagne }\end{array}$ & Espagne & USA & Italie \\
\hline Entreprise & 0 & 3 & 2 & 0 & 1 \\
\hline Université & 2 & 7 & 7 & 1 & 0 \\
\hline \hline Pays & Canada & Mexique & Islande & Australie & TOTAL \\
\hline Entreprise & 1 & 1 & 1 & 0 & 09 \\
\hline Université & 0 & 0 & 0 & 1 & 18 \\
\hline
\end{tabular}

La tendance amorcée l'année précédente, non seulement se confirme, mais laisse apparaître une quasi-parité entre les séjours linguistiques et les stages en entreprise (cf. tableau 7). Les perspectives pour la prochaine année universitaire 1998-1999 révèlent que seuls trois étudiants, sur un total de dix-sept, désirent partir dans une université étrangère. Le département dispose de 10 bourses FACE et 5 bourses LEONARDO d'un montant proche de $2500 \mathrm{FF}$ mensuels pour défrayer les stagiaires. C'est l'exact contraire du premier échange de 1979.

Tableau 7. Stage en entreprise ou en université en 1997-1998

\begin{tabular}{|c|c|c|c|c|}
\hline Pays & $\begin{array}{c}\text { Grande- } \\
\text { Bretagne }\end{array}$ & Espagne & Allemagne & TOTAL \\
\hline Entreprise & 4 & 2 & 1 & 07 \\
\hline Université & 5 & 3 & 1 & 09 \\
\hline
\end{tabular}

Quelles interprétations donner de ces tendances insensibles mais sûres qui s'inversent en deux décennies? Il faut peut-être chercher des essais d'explication dans la structure universitaire elle-même mais aussi dans les changements survenus dans la société tout entière.

\section{La filière LEA dans la structure universitaire}

Établi dans la Faculté des Lettres (dans la même enceinte, les mêmes locaux, animé par le même corps enseignant), on aurait pu craindre que le département LEA renforçât peutêtre, au lieu de la modifier, l'idée que l'étudiant se formait de l'Université ; qu'il y vît une réplique, un double plutôt qu'une entité différente, le percevant comme une extension 
peu claire du département LCE. La présence, en nombre important, d'enseignants de LCEanglais (il n'y a toujours que deux postes statutaires en LEA pour environ 500 étudiants en 1989-90, et environ 300 en 1997-98) joue également dans le sens d'une faible lisibilité. Pourtant rien de tel ne fut constaté pendant la première décennie. Tous les indicateurs convergeaient alors pour indiquer une réussite éclatante: variété des origines géographiques, scolaires des étudiants, facilité et diversité de l'insertion professionnelle, comme en témoignent les résultats de l'enquête de 1986. Trois étudiants sur quatre - $75 \%$ de l'effectif - trouvent alors un emploi correspondant à la vocation affichée de la filière.

Tableau 8. Résultats 1975-1985

\begin{tabular}{|c|c|c|c|}
\hline Enseignement & Entreprise & Administration & $\begin{array}{c}\text { Autre } \\
\text { Exploitant agricole }\end{array}$ \\
\hline 11 & 26 & 13 & 02 \\
\hline $21,50 \%$ & $50 \%$ & $25 \%$ & $3,38 \%$ \\
\hline
\end{tabular}

Population interrogée: 52

Les moyens matériels, tant internes qu'externes, sont éclairants eux aussi. L'ouverture d'une section de Langues étrangères appliquées n'a pas généré de ligne de crédit ni de dotations budgétaires à la mesure des besoins requis pour une telle formation. Si l'on met en regard les seules conditions pédagogiques des LEA, IUT-Techniques de commercialisation (BOEN 1997 : 427) et BTS assistant secrétaire trilingue par exemple, force est de constater qu'elles sont incomparables.

Tableau 9. Comparaison conditions pédagogiques IUT, BTS et LEA

\begin{tabular}{|c|c|c|}
\hline $\begin{array}{c}\text { IUT } \\
\text { Techniques de } \\
\text { Commercialisation }\end{array}$ & \begin{tabular}{|c} 
BTS \\
Assistant secrétaire \\
trilingue
\end{tabular} & LEA \\
\hline $\begin{array}{l}\text { Les travaux dirigés sont organisés en groupes de } 28 \\
\text { étudiants au maximum. } \\
\text { La taille des groupes de TP correspond à la moitié de celle } \\
\text { des groupes de TD. Toutefois, certains TD et TP peuvent [...] } \\
\text { pour des raisons d'installations particulières, comporter des } \\
\text { effectifs plus restreints. }\end{array}$ & \begin{tabular}{|l} 
Effectif : à $22+1$, la \\
classe est dédoublée \\
Actions \\
professionnelles : \\
3 heures \\
consécutives/ \\
semaine : \\
classe dédoublée
\end{tabular} & $\begin{array}{l}\text { Aucune } \\
\text { norme }\end{array}$ \\
\hline
\end{tabular}

Une composante pauvre, sans vrais moyens d'accompagnement a relevé le défi d'attirer, pour ne pas dire fixer et fidéliser étudiants et professionnels de qualité. La filière a réussi ce tour de force tout au long de la première décennie. De fortes potentialités existaient au départ. Les taux de réussite à l'examen, la concordance entre le titre (ou diplôme) et le 
poste obtenu à la sortie comme le montre le résultat des enquêtes sur l'insertion professionnelle des étudiants en 1985 et 1994, en témoignent. marketing, le management, l'économie et l'histoire qui souvent leur inspirent un sujet pour leur mémoire. L'immersion universitaire ininterrompue qui s'ensuit tendrait à expliquer une certaine propension des étudiants de LEA à se réorienter, à leur retour, vers LCE où certains s'inscrivent à la préparation des concours de recrutement de l'enseignement secondaire. L'augmentation du nombre de places offertes à ces derniers pouvait à juste titre retenir leur attention. Certains, moins nombreux, optent pour la filière longue : DESS dans une autre université ou, fait plus rare, sur place DEA puis thèse en LCE. L'encadrement professoral (un enseignant pour 15 ou 18 étudiants en moyenne là-bas contre un pour 50 ici) tend à réveiller le goût des stagiaires pour la réflexion et le cheminement personnel. L'influence est parfois explicitement reconnue. Ainsi une étudiante partie à Charlotte (Caroline du Nord) qui ayant effectué un stage d'entreprise et néanmoins rédigé un mémoire civilisationniste ("The American trade embargo against Vietnam, 1975-1994. The debate For and Against the lifting of the embargo ») en attribue le mérite à un merveilleux professeur d'histoire américaine, comme elle l'indique en exergue à son travail.

Ce retour vers, et donc l'abandon du choix premier de LEA, est renforcé par un facteur extérieur non négligeable: celui de la conjoncture déprimée de l'emploi. Dans la tourmente économique qui perturbe le monde et l'Europe (le travail rare et précaire se généralisant dans la plupart des pays européens en même temps que le laminage de l'ensemble des acquis sociaux), les étudiants redécouvrent avec soulagement la voie des concours du secteur public et de l'enseignement tels qu'ils existent encore en France et s'y engagent avec détermination. 
77 L'enquête sur l'insertion professionnelle des étudiants en 1985 et 1994 révèle, dans une inversion quasi parfaite des chiffres entre 1975-85 et 1987-94, deux décennies très contrastées où les étudiants se sont repliés de l'option professionnelle qu'ils avaient initialement choisie vers le secteur public du fonctionnariat et de l'enseignement (voir tableau 10).

Tableau 10. Résultats 1987-1994

\begin{tabular}{|c|c|c||c|c|c|c|}
\hline Études & IUFM & Enseignement & Administration & Entreprises & Recherche Emploi & $\begin{array}{c}\text { Non } \\
\text { Réponse }\end{array}$ \\
\hline 17 & 08 & 15 & 06 & 39 & 09 & 03 \\
\hline \hline & $=40$, soit $40,81 \%$ & $6,12 \%$ & $40,81 \%$ & $=12$, soit $12,24 \%$ \\
\hline
\end{tabular}

Population interrogée : 93

L'enseignement dépasse la barre des $40 \%$ (2 étudiants sur 5) alors que précédemment (voir supra), il représentait à peine plus de $20 \%$ des choix ( 1 sur 5). On ne peut s'empêcher de noter un parallélisme étroit entre ces chiffres et l'importante augmentation du nombre de postes offerts aux concours d'enseignement durant cette période (politique de recrutement en accordéon dont l'Éducation nationale semble détenir le secret). Le début de la décennie a également coïncidé avec l'ouverture des IUFM dont les modalités de recrutement et de formation ont pu séduire les étudiants de maîtrise LEA.

79 Si l'on additionne aux débouchés générés par l'enseignement ceux des sans-emploi (la mention n'était même pas prévue dans les réponses possibles de l'enquête précédente ), plus de $12 \%$ en 1994, le total passe la barre de $50 \%$, c'est-à-dire qu'au cours des huit années examinées, moins d'un étudiant sur deux s'est dirigé vers les entreprises ou les administrations.

80 S'ajoute peut-être à cette dernière raison, la relative saturation de débouchés locaux pour une filière qui, après être apparue comme nécessaire, a vu se multiplier la concurrence des universités limitrophes. De nombreux départements LEA ont en effet vu le jour; cependant l'argument, qui pèse son poids en période de dépression, ne devrait guère résister à l'analyse dans une économie dynamique. S'il est prématuré de recommencer une enquête similaire à celle de 1994 quatre ans plus tard, il semble pourtant qu'un mouvement nouveau se dessine sous la pression de facteurs extérieurs qui ne peuvent être ignorés.

\section{Conclusion}

81 Ces Travaux d'études et de recherche révèlent deux traits dominants. Le premier est que si la plupart des mémoires obéissent strictement aux exigences formelles qui sont requises dans les directives (présentation, longueur, rédaction, voire choix du thème), ils s'en éloignent en ce qu'ils trahissent une difficulté certaine à identifier une question se posant dans un environnement réel, à la formuler et à en mener à bien l'analyse. Poser un problème précis dans un champ donné ou une entreprise particulière et se diriger vers 
une solution, en combinant rigueur et pertinence de l'observation, est seulement l'apanage des meilleurs.

Le second trait en revanche, qui s'inscrit en opposition radicale du premier, est la facilité spontanée (ou acquise) avec laquelle les étudiants s'adaptent à un environnement en rapide changement. Leur aptitude à saisir au vol les occasions qui se présentent, témoignant d'un réel mimétisme, reste frappante dans les promotions examinées. Transparaît un solide pragmatisme qui puise également aux sources de l'institution universitaire et à celles du monde des entreprises.

Dans les premières années ayant suivi l'ouverture du département LEA, les enseignants ont joué à fond le jeu de la professionnalisation d'autant qu'alors la filière correspondait à de réels besoins dans le pays qui se préparait au marché unique de 1993, où l'idée de « former ensemble une grande Europe » étant encore présente. L'échéance suscitait alors impatience et optimisme. Ce dernier sentiment fut également au rendez-vous lors de la mise sur pied des échanges européens, bien qu'avec des perspectives plus immédiatement utilitaristes : c'était le meilleur biais possible pour inciter les étudiants à envisager un séjour à l'étranger. Mais très tôt ceux-ci (et pourquoi pas, leurs familles aussi) se sont rendu compte que les aides à la mobilité diminuaient sensiblement et que la concurrence pour leur obtention se faisait plus âpre. En somme, la carte européenne n'était plus un aussi bon atout pour les études qu'on voulait le laisser croire. À ce moment-là, les sirènes de l'Éducation nationale ont attiré les navigateurs sans destination précise, mais lorsque l'éventail des postes a commencé de se refermer, les regards se sont à nouveau portés vers l'extérieur. Quand le service public est accueillant, qu'il ouvre l'accordéon de ses emplois, alors les choix initiaux : LEA-formation pratique sont abandonnés à son profit ; mais qu'il cède à un malthusianisme chronique, se figeant dans une posture d'inaccessibilité, alors les efforts des étudiants se reportent sur le monde des entreprises et l'énergie qu'ils déploient pour être à la hauteur des attentes minimales devient parfois surprenante. Ceux-là savent fort bien que les emplois dans le secteur privé signifient pour eux affronter la concurrence mondiale et donc autant que faire se peut, en connaître les tenants et les aboutissants. Ils sentent que désormais la notion d'Europe est un terme dépassé, peut-être avant d'avoir été jamais atteint.

Quelle était la vraie échéance fondatrice: celle de 1992 dite politique, économique et sociale ; celle de 2002, exclusivement financière (il n'est qu'à se référer à l'orchestration de la campagne sur l'euro pour s'en persuader); ou bien la prochaine encore plus contraignante pour celui qui doit affronter le marché de l'emploi ? Le comportement des étudiants semble indiquer qu'à défaut de percevoir une Europe lisible à l'horizon de leur vie adulte, ils préfèrent encore anticiper le mouvement de compétition. Dans une économie mondialisée, ultra-libérale où les notions de pays et de frontières sont rendues obsolètes, les plus lucides sentent qu'ils n'ont guère d'autre option que le travail en entreprise et qu'une pré-expérience professionnelle, internationale sous peine d'être récusée, est un atout indispensable (voir l'enquête de 1991-92 auprès des entreprises). Ainsi pourrait s'interpréter l'attitude de ces jeunes gens et jeunes filles qui à nouveau se dirigent vers les stages d'entreprise et la formation professionnalisante : ils n'ignorent pas que, pour retenir quelques chances dans le monde aléatoire de l'emploi, l'accumulation précoce des expériences est critère de sélection, donc impérieuse nécessité. En même temps, ils témoignent de leur courage et de leur esprit d'aventure, manifestant leur confiance dans un avenir pourtant fort incertain. 


\section{BIBLIOGRAPHIE}

\section{Textes officiels}

Arrêté du 28 janvier 1976 habilitant l'Université de Limoges à délivrer des diplômes nationaux de l'enseignement supérieur.

Arrêté du 27 janvier 1981 relatif à la dénomination nationale de licence et maîtrise de Langues Etrangères Appliquée.

« Licence et maîtrise de LEA », Journal officiel de la République française, 6762 et 6764, 4 mai 1997.

« Programmes pédagogiques nationaux des IUT, Techniques de Commercialisation ». 1997 BOEN 17, hors série, volume 4, 411-428, 18 déc. 1997.

Ministère de l'Éducation nationale, de la Recherche et de la Technologie. 1997a. Brevet de Technicien supérieur. Assistant secrétaire trilingue, Collection Horaires/Objectifs/Programmes/ Instructions, Arrêté du 3 septembre 1997 et annexes.

Ministère de l'Éducation nationale, de la Recherche et de la Technologie. 1997b. Direction de l'Évaluation et de la Prospective. Panorama du système éducatif français, Présentation de Claude Allègre et Ségolène Royal, septembre.

\section{Documents LEA-Limoges}

Note à l'attention des chefs d'entreprise, 1er février 1977.

Anonyme. 1980. Rapport sur l'enseignement des LEA à l'UER des Lettres de Limoges, 31 janvier 1980.

Département de Langues Étrangères Appliquées. Programme des Études, 1987-88.

Dumont, Maurice. 1995. « Maîtrise de LEA : Instructions relatives au mémoire ».

Langues Étrangères Appliquées. 1998. Maîtrise 97-98, option affaires et commerce, (AnglaisAllemand, Anglais-Espagnol, Allemand-Espagnol), Programmes des études.

Travaux d'Études et de Recherches ou Mémoires (131 TER consultés), années 1977-78 à 1996-97.

\section{Enquêtes}

AALEA-Limoges. 1995. Association des Anciens Étudiants de Langues Etrangères Appliquées de Limoges. Annuaire 1995 des anciens étudiants LEA-Limoges, Limoges.

Devenir des étudiants ayant obtenu un diplôme de langues étrangères appliquées à l'université de Limoges entre 1975 et 1981. 1982. Limoges : ONISEP.

Faculté des Lettres. 1987. Langues Étrangères Appliquées en Limousin. Limoges :ONISEP, avril.

Gontier,J.L. (dir.). 1991-1992. « Perception de la filière Langues Etrangères appliquées par les entreprises du Limousin », rapport réalisé par un groupe d'étudiants en maîtrise de Langues Etrangères Appliquées, Limoges.

Ndoumou, M. (assistante au CROUS de Limoges). 1980.« Les étudiants en lettres de Limoges de l'année 1978-79. Origine géographique et sociale. Motivations et objectifs ». Conditions d'études. 
Renaudin,Marie-Pierre,Simone Simon (étudiants en Sciences de l'éducation) et Pierrette Arnaud, directrice du département de Psychologie, sociologie et Sciences de l'éducation.1994.« Langues Etrangères Appliquées à l'université de Limoges. Cursus universitaire et insertion professionnelle des étudiants issus de LEA de 1986-87 à 1992-93 ». Université de Limoges.

\section{ANNEXES}

Quelques exemples de titres de Travaux d'études et de recherche (TER)

Agriculture française ou anglaise :

1981 : Farming in Hampshire

1982 : British agriculture

1983 : The stuffed liver (sic ! foies gras) \& truffle fairs (sic !) in Thiviers (Dordogne)

1984 : The agriculture in Creuse and its problems

1985 : The Limousine breed

1986 : Young farmers in Limousin

1987 : The Limousin Herd Book

1987 : British agriculture \& EEC problems

1992 : British agriculture, British farmers and the common agricultural policy in the 1970's.

\section{Local industry :}

1988 : Stoke and Limoges : Two centres of pottery

1993 : Luxury Ceramic Ware industry in North-Staffordshire

\section{Portsmouth :}

1980 : City of Portsmouth Today \& Tomorrow

Industrial evolution of Portsmouth : Main industrial sectors in the area

The industrial development of Portsmouth from the 19th century up to the present days 1981 : The geographical evolution of Portsmouth and its history

The planning process in Portsmouth since the second World War

The development of Portsmouth Harbour

1982 : Portsmouth Polytechnic

Being a student in Portsmouth

Portsmouth local government

Everyday economic life in Portsmouth

Everyday social life in Portsmouth

1983 : Unemployment in Portsmouth

1983 : The newspaper in Portsmouth : the News 
1988 : Portsmouth, birthplace of Australia

1982 : The Christian worships in Portsmouth

1988 : Come on, Pompey (Portsmouth football team )

1982 : Tourism in Portsmouth \& Southsea, a seaside \& historic city

1981 : Mary Rose-its fate \& future

1982 : A treasury in Portsmouth - HMS Victory

1992 : Tourism development in the Isle of Wight

Manchester :

1988 : Manchester City Centre Development. Council housing in Manchester

1989 : Greater Manchester-Preserving \& Improving Environment in an industrial area

1991 : Manchester jewry

1993 : How does Manchester airport run its communication?

Housing problems in Manchester

Bath :

Bath during the Roman occupation

Bath : the fortunes of the Theatre Royal against their social and historical background

Evolution of housing in Georgian Bath

Économie, technologie et industrie :

North sea oil \& economic contribution to Great Britain's development

A conservative area threatened by the oil industry

1984 : The Channel tunnel case

1994 : The Pros and Cons of the Channel tunnel

1987 : British technology

1987 : The High-Tech new Wave

1987 : Free Zones and enterprise zones : Oases for British and French economy

1995 : VFM : the logistics function : minimizing costs and improving customer service.

\section{Questions sociales :}

1983 : Poverty \& Inequality in Great-Britain

1992 : Poverty in Great Britain

1985 : Inside the British police

British police : the end of tradition

1983 : Her Majesty's prisons

1983 : The effects of industrialization on female condition in Great Britain

1984 : Housewife 
1988 : Incest : facing the unthinkable

1988 : Voluntary organization for the elderly

1986 : Post-war youth subcultures

1988 : Youth tribes in Great Britain

1987-88: The introduction of the community charge or poll tax in England and Wales

(1987-1990)

1992 : The community charge : its economic and social effect

1987 : The health service

1987 : British politics and the NHS

1992 : Crisis in the National Health Service

\section{L'institution universitaire :}

1981 : Portsmouth Polytechnic

1981 : Being a student in Portsmouth

1989 : Will British students do better off with « top-up loans »? An analysis of different styles of student aid

1990 : British universities : The end of government funding, at what cost?

1990 : Top-Up loans for students : A life without grants

1994 : What do you mean by student's unions?

1997 : UNCC : a major economic incentive for the Charlotte area

Presse, média, publicité :

1982 : The newspapers in Portsmouth : The News

British press : The Independent

1982 : An Independent local radio : Radio Victory

1982 : R.C.O. or the story of a decentralized radio station

1987 : Irish local radio

1980 : Advertising media

1986 : Freedom of the media

1989 : Study of a publicity program for Bestobell Mobrey

\section{Politique :}

1981 : Informal colonialism in Anglo-Portuguese relations

1983 : The Falklands Islands (The war, the consequences, the British reaction)

1991 : Doing business with the Middle East : Difficulties and Options

1987 : The trade unions \& British politics 
1990 : A resignation that casts doubts on thatcherism \& British economy (la démission de Mr Nigel LAWSON, Chancellor of the Exchequer à propos de EMS, European Monetary System)

1986 : The provisional IRA

1992 : Northern Ireland : A study of the problem from its origin to the present situation 1994 : The British policy in Northern Ireland : Keeping the peace or making war?

1997 : The American trade embargo against Vietnam, 1978-1994. The debate for and against the lifting of the embargo

Cas particulier d'un étudiant de Portsmouth en année « sabbatique » ou « sandwich » à Limoges : The French debate over West German rearmament (1945-1954) abordant une question qui lui a valu le Henry Walton Memorial Prize (for an outstanding dissertation in the area of German studies)

\section{NOTES}

1. Deux enquêtes avaient été effectuées précédemment avec des taux de réponses croissant parallèlement aux effectifs, de 1975 à 1986. L'enquête de 1982 obtenait $65 \%$ de réponses, celle de 1986, 58 \% et celle de 1992-93 seulement $25 \%$.

\section{RÉSUMÉS}

L'auteur se propose, à travers l'étude des mémoires et des stages de maîtrise, obligatoires pour l'obtention du diplôme, de tester la visibilité de la formation pratique, appliquée, proposée par LEA. L'étude sera menée à partir des travaux des vingt dernières années, de 1978, première promotion de maîtrise, jusqu'à 1997, dernière année en date. Elle visera à mettre en évidence la ventilation selon les champs culturel, social, économique, politique... des différents sujets; le poids des différentes langues; l'incidence des liens avec les établissements universitaires étrangers. Les éléments ainsi réunis seront contrastés avec l'objectif déclaré de la filière LEA, dans son habilitation puis dans les modifications qu'elle y a apportées au fil de deux décennies.

This article addresses the practical dimension of the LEA program through a study of the theses and vocational periods required to obtain the maitrise degree. The theses of the last twenty years, from 1978, the opening year, to 1997, the latest to date- will serve as a basis. The aim is to identify the kind of topics chosen in the respective cultural, social, economic and political fields. Also stressed is the salience of each language learnt as well as the links with European institutions. The various elements thus examined are contrasted with the founding objectives, and the changes that have been made, over the last two decades. 
INDEX

Keywords : LEA, LEA theses, practical training, TER, training course, vocational training Mots-clés : formation pratique, formation professionnelle, LEA, maîtrise, stage, TER

\section{AUTEUR}

\section{ÉMILIE CLAUZURE}

Agrégée d'anglais, Émilie Clauzure est maître de conférences à la faculté des Lettres, Université de Limoges. Elle a soutenu en 1996 une thèse de doctorat intitulée « Political Correctness :

l'affrontement idéologique au sein des universités américaines 1984-1994. » Elle s'intéresse à l'enseignement supérieur (américain), sa structure, son fonctionnement ainsi qu'aux aspects de la langue, en particulier la néologie et la terminologie. clauzure2@free.fr 